\title{
Padrões espaciais de mortalidade e diferenciais sócio-econômicos na cidade do Rio de Janeiro
}

\author{
Mortality spatial patterns and socioeconomic \\ differences in the city of Rio de Janeiro
}

Simone M. Santos 1

Claudio P. Noronha 2

\footnotetext{
1 Departamento de Informações em Saúde, Centro de Informação Científica e Tecnológica, Fundação Oswaldo Cruz. Av. Brasil 4365, Rio de Janeiro, RJ 21045-900, Brasil. smsantos@malaria.procc. fiocruz.br

2 Coordenação de Programas de Epidemiologia, Superintendência de Saúde Coletiva, Secretaria Municipal de Saúde do Rio de Janeiro. Rua Afonso Cavalcanti 455 sala 828 , Rio de Janeiro, $R$ 20211-110, Brasil. cnoronha@pcrj.rj.gov.br
}

\begin{abstract}
Health situation analysis has been used to understand complex health processes and to contribute to decision-making in public policies. This paper analyzes the spatial relationship between mortality patterns in the city of Rio de Janeiro (1996-1998) and socioeconomic profiles of neighborhoods. Mortality data were collected from the Municipal Health Department and population data were obtained from the National Census Bureau (IBGE) (1991 and 1996). Neighborhoods were aggregated through the K-means cluster method into 4 homogeneous socioeconomic groups, and the mortality indicators distribution was analyzed by different strata. General mortality, circulatory disease mortality, mortality from poorly defined causes, and mortality from violent causes all displayed large differences among socioeconomic groups. The spatial pattern of the socioeconomic strata allowed the identification of different living conditions, which involved specific mortality patterns. Higher socioeconomic strata presented low mortality figures for all causes.
\end{abstract}

Key words Spatial Analysis; Mortality; Social Indicators

Resumo A análise de situação de saúde tem sido usada para compreender a complexidade dos fenômenos que envolvem a saúde e contribuir para a tomada de decisão em relação a políticas públicas. Este trabalho analisa as relações espaciais entre os padrões de mortalidade da cidade do Rio de Janeiro (1996-1998) e o perfil sócio-econômico dos bairros, criados com base nos dados do IBGE (1991 e 1996). Mediante o método de aglomeração K-means, constituíram-se quatro grupos sócio-economicamente homogêneos e posteriormente analisou-se a distribuição dos indicadores de mortalidade nos diferentes estratos. A mortalidade geral, a por doenças circulatórias, a por causas mal definidas e a por causas externas foram as que apresentaram maiores diferenças entre os grupos sócio-econômicos. O padrão espacial dos estratos sócio-econômicos retratou as diversas condições de vida dos cariocas implicando padrões de mortalidade específicos. Os estratos sócio-economicamente mais favorecidos apresentaram um perfil de baixa mortalidade para todas as causas.

Palavras-chave Análise Espacial; Mortalidade; Indicadores Sociais 


\section{Introdução}

A análise de dados espaciais tem apresentado rápido crescimento dentro da saúde pública, constituindo-se em uma área de conhecimento que busca a integração de um conjunto de saberes de outras disciplinas, com enorme contribuição a diversos estudos, desde aqueles que visam a produção de subsídios às estratégias de intervenção de políticas públicas àqueles voltados à exploração etiológica dos eventos em saúde.

Se a doença é uma manifestação do indivíduo, as condições de vida são manifestação do lugar (Barcellos, 2000). A relação entre os lugares e a produção de doenças resultam de um acúmulo de situações históricas, ambientais e sociais. Para o diagnóstico das condições de vida, é necessária a construção de indicadores que detectem os diferenciais de risco à saúde oriundos desta diversidade social e ambiental. (Akerman et al., 1994; Di Villarosa et al., 1990). Estes indicadores devem permitir a identificação de diferentes situações de risco, possibilitando o direcionamento das ações de saúde, de modo mais específico, aos eventos que se pretende prevenir e controlar.

O uso do geoprocessamento na área de saúde tem sido facilitado pelo amplo acesso a bases de dados epidemiológicos e pela disponibilização de ferramentas cartográficas e estatísticas computadorizadas. Estes avanços possibilitam a confecção rápida de mapas temáticos que podem contribuir para a formulação de hipóteses a respeito da distribuição espacial de agravos à saúde e sua relação com indicadores sócio-econômicos (Rojas et al., 1999).

Os Sistemas de Informação Geográfica (SIG) permitem a integração e combinação de dados cartográficos e seus atributos oriundos de diferentes fontes de dados (Maguirre et al., 1991). A incorporação desta ferramenta às técnicas epidemiológicas contribui para a análise de informações acerca da mortalidade e condições sócio-econômicas das populações que ocupam diferentes territórios de uma cidade. Alguns estudos têm sido realizados em capitais brasileiras, procurando associar indicadores sócioeconômicos à ocorrência de agravos à saúde através de diferenciais intra-urbanos (Akerman et al., 1994; Freitas et al., 2000; Lima \& Ximenes, 1998; Paim et al., 1999). A maioria desses estudos têm demonstrado que populações socialmente desfavorecidas apresentam índices mais altos para diversas causas de morte, sejam elas doenças crônicas, infecciosas ou externas. No Município do Rio de Janeiro, os estudos ecológicos que utilizam técnicas de aná- lise espacial, têm tido enfoque na mortalidade por causas externas, na mortalidade infantil e nas condições de vida e nascimento. Estes têm reiterado a importância da desigualdade e os efeitos da privação social e material sobre a população do município. A maior parte destes estudos utilizaram as Áreas Programáticas ou as Regiões Administrativas como unidades espaciais de referência (d'Orsi \& Carvalho, 1998; Souza et al., 1997; Szwarcwald et al., 1999), havendo carência de estudos que analisem o padrão de indicadores nos bairros e de causas específicas de mortalidade que contribuem com maior importância na mortalidade geral.

Nos estudos espaciais, as unidades de análise definem a escala de observação dos fenômenos estudados, limitando as interações que podem ser captadas (Barcellos \& Bastos, 1996). Por sua vez, a escolha da escala de observação é limitada pela disponibilidade das unidades espaciais de referência nos sistemas de informações utilizados (Barcellos \& Santos, 1997). Especificamente, para o Município do Rio de Janeiro, a referência dos dados de mortalidade aos bairros foi disponibilizada somente a partir de 1996.

Os indicadores da situação de saúde dos cariocas oriundos das estatísticas de mortalidade de 1998 (SMS-RJ, 1999) mostram que as doenças do aparelho circulatório são as responsáveis pela maior parte das mortes na cidade $(30,4 \%)$, sendo seguidas pelas neoplasias $(14,3 \%)$, pelas causas externas $(11,8 \%)$, pelas doenças do aparelho respiratório $(11,5 \%)$ e pelas causas mal definidas (10,7\%). Este perfil de mortalidade proporcional reflete as características das condições de vida na cidade, onde sobressaem as doenças crônicas, a violência social, as deficiências da rede de serviços de saúde, dentre outras. Em relação às doenças crônicas, elas também refletem as mudanças no perfil epidemiológico da população carioca, em que se destaca o aumento da população idosa conseqüente da elevação da expectativa de vida e da queda nas taxas de mortalidade. Esta característica não se apresenta de forma homogênea na cidade; pelo contrário, existem diferenças importantes no perfil etário das diversas regiões geográficas, o que traz, como conseqüência imediata, a necessidade de padronizar as taxas de mortalidade de cada área para garantir comparabilidade na análise das diferenças internas (Noronha et al., 1998).

O objetivo deste trabalho é analisar as relações dos padrões espaciais de mortalidade dos 153 bairros da cidade do Rio de Janeiro, no período de 1996 a 1998, com o perfil sócio-econômico dos bairros e nas microáreas sócio-economicamente homogêneas que compõem o município. 


\section{Materiais e métodos}

Neste trabalho foi utilizado um Sistema de Informações Geográficas (SIG) com bases cartográficas de setores censitários referentes ao censo demográfico de 1991, bem como dos limites dos bairros, criadas pela equipe do projeto SIG/Fundação Oswaldo Cruz (FIOCRUZ), desenvolvido no Departamento de Informações em Saúde (Centro de Informação Científica e Tecnológica - CICT, FIOCRUZ). Em 1991, o Município do Rio de Janeiro era formado por cerca de 6.400 setores censitários, agrupados em 153 bairros. Para as análises estatísticas foram excluídos nove bairros com menos de 3.000 habitantes, conferindo maior estabilidade aos indicadores.

A escolha dos indicadores sócio-econômicos baseou-se nos estudos de Carvalho et al. (1997) e de d'Orsi (1996). O primeiro, analisou cerca de cinqüenta indicadores oriundos do censo demográfico de 1991 segundo os temas: saneamento, ocupação de terreno, escolaridade, domicílio, demografia e renda. Através da análise de componentes principais e do poder de explicação da variância observada, foram definidos indicadores que possibilitam boa diferenciação das Áreas Programáticas no Município do Rio de Janeiro e sua Região Metropolitana. O segundo, relativo à distribuição espacial da mortalidade infantil e condições de nascimento no Município do Rio de Janeiro, selecionou cinco indicadores para caracterizar grupos de bairros sócio-economicamente homogêneos, com metodologia semelhante. Neste último estudo foi observado que o uso de indicadores que discriminem melhor as áreas faveladas e as áreas com renda alta, ambos fortemente correlacionados com a escolaridade, são importantes para diferenciar microáreas no município.

Os indicadores sócio-econômicos utilizados foram construídos a partir dos dados do censo demográfico de 1991 (IBGE, 1994), agrupados por bairro. Os indicadores sócio-econômicos analisados foram: proporção de casas, como um padrão de construção que expressa a verticalidade da ocupação residencial; proporção de domicílios com esgoto ligado à rede geral, que reflete as condições de saneamento; proporção de domicílios subnormais, a qual evidencia a precariedade de infra-estrutura urbana e de construção irregular das moradias (favelas e loteamentos irregulares); proporção de chefes de família com renda superior a 10 salários, utilizada como expressão da concentração de renda; e, proporção de domicílios alugados, que destaca a distribuição dos grupos sociais intermediários (classe média), pois os residentes em imóveis irregulares têm posse não legalizada, enquanto a classe alta tem propriedade de seus imóveis em razão de seu melhor padrão econômico.

Os bairros que compõem o município foram caracterizados segundo o perfil desses indicadores através de análise classificatória multivariada de agrupamento pelo método $K$-means (Hartigan, 1975), no pacote estatístico SPSS (SPSS Incorporation, 1997), formando quatro grupos distintos, que delimitaram diferentes microáreas sócio-econômicas. A classificação multivariada, também denominada análise de cluster, é um conjunto de procedimentos que visa identificar grupos relativamente homogêneos de casos (bairros, neste estudo) baseados em características selecionadas (variáveis sócio-econômicas, neste estudo). Estes agrupamentos são definidos por critérios fundamentados em distâncias. Neste estudo foi utilizado o método não hierárquico K-means, que emprega a distância Euclidiana para definir o centro dos grupos. A partição realizada busca diminuir a variância intragrupos e maximizar a variância intergrupos. $\mathrm{O}$ valor relativo da estatística $\mathrm{F}$ de variância permite demonstrar a contribuição de cada variável na separação dos grupos.

Os indicadores de mortalidade foram calculados a partir de dados do Sistema de Informações sobre Mortalidade, do Ministério da Saúde, coletados pela Coordenação de Epidemiologia da Secretaria Municipal de Saúde, classificados segundo a Décima Revisão da Classificação Estatística Internacional de Doenças e Problemas Relacionados à Saúde (OMS, 1995), e dados populacionais oriundos da Contagem Populacional, de 1996 (IBGE, 1998). O georreferenciamento dos óbitos foi efetuado através da variável bairro de residência, que consta na declaração de óbito. Os indicadores criados foram: a taxa de mortalidade geral, a taxa de mortalidade por doenças infecto-contagiosas, a taxa de mortalidade por neoplasias, a taxa de mortalidade por doenças circulatórias, a taxa de mortalidade por causas mal definidas, a taxa de mortalidade por doenças respiratórias e a taxa de mortalidade por causas externas, todas ajustadas pela estrutura etária da população do município, em faixas de cinco em cinco anos e mais de 70 anos.

Para analisar mais detalhadamente a variabilidade dos indicadores, foi calculada a correlação bivariada de Pearson, visando explicitar relações que ficam suavizadas na comparação das médias entre os grupos de bairros. Da mesma forma, são apresentados mapas das princi- 
pais taxas de mortalidade conforme categorias de desvio padrão de suas distribuições por bairro.

\section{Resultados}

\section{Caracterização sócio-econômica de cada grupo de bairros}

Os indicadores sócio-econômicos de cada grupo de bairros podem ser observados na Tabela 1 e sua distribuição é apresentada na Figura 1. Os bairros que compõem cada grupo estão listados na Tabela 2. As principais características de cada grupo são descritas abaixo.

O grupo A é o grupo de melhor renda, mais verticalizado e de maior urbanização. Apresenta a menor proporção de casas e de domicílios subnormais. É o grupo de maior proporção de chefes de família com renda mensal maior ou igual a dez salários mínimos, maior proporção de domicílios ligados à rede de esgoto (96\%) e de domicílios alugados. Este grupo abrange a quase totalidade dos bairros da zona sul e os bairros mais tradicionais da zona norte da cidade como, por exemplo, Tijuca, Vila Isabel.

O grupo B concentrou o maior número de bairros (64) e evidencia uma proporção de domicílios alugados e domicílios ligados à rede de esgoto, semelhantes aos do Grupo A. A proporção de domicílios subnormais é próxima à média do município e apenas $7,8 \%$ dos chefes de família têm renda mensal maior ou igual a dez salários mínimos, quase a metade da média do município. Os bairros que compõem este grupo abrangem áreas dos antigos subúrbios cariocas.
O grupo C apresenta a maior proporção de domicílios subnormais (57\%), com grande proporção de casas e pequena proporção de domicílios alugados, nos quais apenas $6 \%$ dos chefes de famílias têm renda mensal maior ou igual a dez salários mínimos. Este grupo concentrou a maior parte das favelas. Ainda assim, é o mais heterogêneo internamente, pois abrange alguns bairros que contêm um mosaico de favelas e áreas urbanas mais estruturadas como, por exemplo, a Gávea, que incorporou as informações da favela da Rocinha, e Bonsucesso, que incorporou o Complexo da Maré e o Morro do Alemão.

O grupo D possui características menos urbanizadas, em que apenas $6 \%$ dos domicílios são ligados à rede geral de esgoto com a maior proporção de casas (90\%). Apresenta a menor proporção de domicílios alugados, poucos domicílios subnormais e a menor proporção de chefes de família com renda mensal maior ou igual a dez salários mínimos.

\section{Caracterização dos indicadores de mortalidade de cada grupo de bairros}

O padrão de mortalidade das populações residentes nos diferentes grupos de bairros (Tabela 3 ) é descrito, a seguir.

O grupo A mostra as menores taxas de mortalidade geral e as menores taxas de mortalidade específicas por doenças circulatórias, respiratórias, por causas mal definidas e por causas externas. Para todos os indicadores de mortalidade, a média apresentada foi menor do que a média para o município.

Tabela 1

Perfil dos grupos de bairros segundo médias e valor da razão variância entre/intra-grupos (F) dos indicadores sócio-econômicos. Rio de Janeiro, 1991.

\begin{tabular}{|c|c|c|c|c|c|c|}
\hline \multirow[t]{2}{*}{ Indicador sócio-econômico* } & \multicolumn{4}{|c|}{ Média do indicador no grupo } & \multirow[t]{2}{*}{ Média Total } & \multirow[t]{2}{*}{$\mathbf{F}$} \\
\hline & A & B & C & D & & \\
\hline Proporção de casas & 23,84 & 69,08 & 82,35 & 90,11 & 62,20 & 159,30 \\
\hline Proporção de domicílios subnormais & 4,60 & 13,62 & 57,23 & 13,26 & 17,24 & 101,80 \\
\hline $\begin{array}{l}\text { Proporção de domicílios com esgoto } \\
\text { ligado a rede geral }\end{array}$ & 96,01 & 88,81 & 70,05 & 6,10 & 75,47 & 284,30 \\
\hline Proporção de domicílios alugados & 29,25 & 29,01 & 17,60 & 16,36 & 25,55 & 19,13 \\
\hline $\begin{array}{l}\text { Proporção de chefes de família } \\
\text { com renda mensal maior ou igual } \\
\text { a } 10 \text { salários mínimos }\end{array}$ & 33,20 & 7,80 & 5,79 & 4,99 & 13,79 & 64,20 \\
\hline Número de bairros & 38 & 64 & 20 & 22 & $144^{\star \star}$ & \\
\hline
\end{tabular}

* Todos indicadores apresentaram diferenças significativas com $p<0,000$.

** Foram excluídos nove bairros que possuíam menos de 3.000 residentes. 


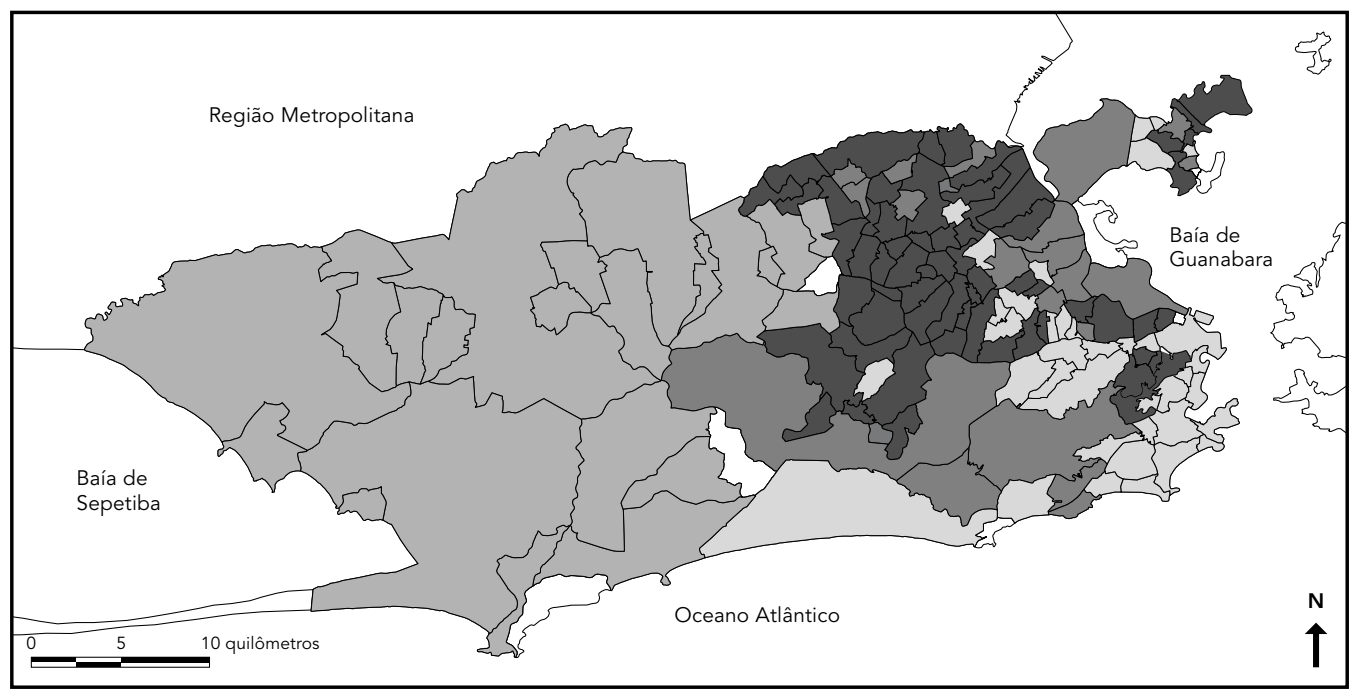

Grupos de bairros conforme condições sócio-econômicas

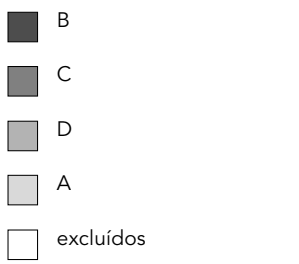

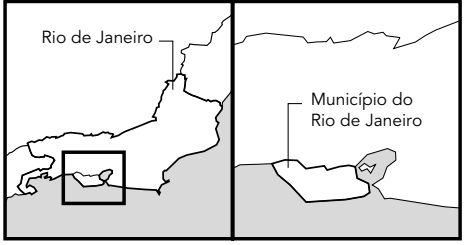

O grupo B obteve as maiores taxas de mortalidade geral e as maiores taxas de mortalidade específica por neoplasias, doenças circulatórias, respiratórias e por causas externas.

O grupo $\mathrm{C}$ apresenta a maior taxa de mortalidade específica por doenças infecto-contagiosas. As demais taxas de mortalidade estão próximas da média do município. Observa-se que este grupo expõe maior variabilidade interna para todas as taxas, expressadas pela amplitude dos intervalos de confiança das taxas médias.

O grupo D obteve a maior taxa de mortalidade por causas mal definidas e a menor taxa de mortalidade por doenças infecto-contagiosas e neoplasias. As demais taxas encontramse em torno da média geral do município. Este grupo abrange a zona oeste com muitos bairros de baixa ocupação e alguns, um pouco melhor estruturados, tais como a Vila Militar e Vargem Grande.

\section{Correlações bivariadas entre os indicadores}

Conforme apresentado na Tabela 4, destacamse as observações descritas a seguir.

Nota-se que a proporção de casas está inversamente relacionada à proporção de chefes com renda maior ou igual a 10 salários mínimos, à proporção de domicílios com esgoto ligado à rede geral e de domicílios alugados e diretamente relacionada à proporção de domicílios subnormais.

As taxas de mortalidade específicas são fortemente correlacionadas entre si e com a taxa de mortalidade geral, refletindo as suas participações na composição da mortalidade geral. A taxa de mortalidade geral tem relação direta com a proporção de casas e inversa com a renda. Na Figura 2, pode-se observar sua distribuição. Este mesmo padrão é apresentado pela mortalidade por doenças circulatórias.

A taxa de mortalidade por causas externas tem relação direta com a proporção de casas e 
Bairros que compõem cada grupo sócio-econômico. Rio de Janeiro, 1991.

\begin{tabular}{|c|c|c|c|}
\hline Grupo A & Grupo B & Grupo C & Grupo D \\
\hline Andaraí & Abolição & Acari & Bangu \\
\hline Barra da Tijuca & Água Santa & Alto da Boa Vista & Barra de Guaratiba \\
\hline Botafogo & Anchieta & Barros Filho & Camorim \\
\hline Cachambi & Anil & Bonsucesso & Campo dos Afonsos \\
\hline Catete & Bancários & Caju & Campo Grande \\
\hline Centro & Benfica & Colégio & Cidade Universitária \\
\hline Cidade Nova & Bento Ribeiro & Costa Barros & Cosmos \\
\hline Copacabana & Brás de Pina & Galeão & Deodoro \\
\hline Cosme Velho & Cacuia & Gávea & Grumari \\
\hline Engenho da Rainha & Campinho & Inhaúma & Guaratiba \\
\hline Flamengo & Cascadura & Itanhangá & Inhoaíba \\
\hline Glória & Catumbi & Jacaré & Jardim Sulacap \\
\hline Grajaú & Cavalcanti & Jacarepaguá & Joá \\
\hline Higienópolis & Cidade de Deus & Manqueira & Magalhães Bastos \\
\hline Humaitá & Cocotá & Manguinhos & Paciência \\
\hline Ipanema & Coelho Neto & Parada de Lucas & Padre Miguel \\
\hline Jardim Botânico & Cordovil & Pitangueiras & Pedra de Guaratiba \\
\hline Jardim Guanabara & Curicica & Ramos & Realengo \\
\hline Lagoa & Del Castilho & Tauá & Recreio Bandeirantes \\
\hline Laranjeiras & Encantado & Vidigal & Santa Cruz \\
\hline Leblon & Engenheiro Leal & & Santíssimo \\
\hline Leme & Engenho de Dentro & & Senador Camara \\
\hline Maracanã & Engenho Novo & & Senador Vasconcelos \\
\hline Méier & Estácio & & Sepetiba \\
\hline Moneró & Freguesia (Ilha) & & Vargem Grande \\
\hline Pechincha & Frequesia (Jpa) & & Vargem Pequena \\
\hline Portuguesa & Gamboa & & Vila Militar \\
\hline Praça da Bandeira & Gardênia Azul & & \\
\hline Praia da Bandeira & Guadalupe & & \\
\hline Riachuelo & Honório Gurgel & & \\
\hline Ribeira & Irajá & & \\
\hline Rocha & Jardim América & & \\
\hline São Conrado & Jardim Carioca & & \\
\hline São Francisco Xavier & Lins de Vasconcelos & & \\
\hline Tijuca & Madureira & & \\
\hline Todos os Santos & Marechal Hermes & & \\
\hline Urca & Maria da Graça & & \\
\hline Vila da Penha & Olaria & & \\
\hline Vila Isabel & Oswaldo Cruz & & \\
\hline \multirow{27}{*}{ Zumbi } & Paquetá & & \\
\hline & Parque Anchieta & & \\
\hline & Pavuna & & \\
\hline & Penha & & \\
\hline & Penha Circular & & \\
\hline & Piedade & & \\
\hline & Pilares & & \\
\hline & Praça Seca & & \\
\hline & Quintino Bocaiúva & & \\
\hline & Ricardo de Albuquerque & & \\
\hline & Rio Comprido & & \\
\hline & Rocha Miranda & & \\
\hline & Sampaio & & \\
\hline & Santa Teresa & & \\
\hline & Santo Cristo & & \\
\hline & São Cristóvão & & \\
\hline & Saúde & & \\
\hline & Tanque & & \\
\hline & Taquara & & \\
\hline & Tomás Coelho & & \\
\hline & Turiacu & & \\
\hline & Vaz Lobo & & \\
\hline & Vicente de Carvalho & & \\
\hline & Vigário Geral & & \\
\hline & Vila Kosmos & & \\
\hline & Vila Valqueire & & \\
\hline & Vista Alegre & & \\
\hline
\end{tabular}

OBS. Os bairros em negrito foram excluídos por possuírem menos de 3.000 habitantes, para garantir a estabilidade das taxas. Ilha = Ilha do Governador; Jpa = Jacarepaguá. 
Perfil dos grupos de bairros segundo médias e valor da razão variância entre/intra-grupos (F) dos indicadores de mortalidade. Rio de Janeiro, 1996 a 1998.

\begin{tabular}{|c|c|c|c|c|c|c|c|}
\hline \multirow[t]{2}{*}{ Indicador de mortalidade* } & \multicolumn{4}{|c|}{$\begin{array}{l}\text { Média do indicador no grupo } \\
\text { Limites inferior e superior do IC }=95 \%\end{array}$} & \multirow[t]{2}{*}{ Média Total } & \multirow[t]{2}{*}{$\mathbf{F}$} & \multirow[t]{2}{*}{ Significância } \\
\hline & A & B & C & D & & & \\
\hline \multirow[t]{3}{*}{ Taxa de mortalidade geral } & 706,56 & 1013,63 & 985,15 & 913,45 & 913,33 & \multirow[t]{3}{*}{5,05} & \multirow[t]{3}{*}{0,002} \\
\hline & 625,54 & 905,40 & 761,05 & 732,64 & 845,44 & & \\
\hline & 787,57 & 1121,86 & 1209,25 & 1094,25 & 981,23 & & \\
\hline \multirow{3}{*}{$\begin{array}{l}\text { Taxa de mortalidade por doenças } \\
\text { infecto-contagiosas }\end{array}$} & 44,76 & 56,06 & 59,13 & 44,45 & 51,73 & \multirow[t]{3}{*}{2,54} & \multirow[t]{3}{*}{0,059} \\
\hline & 37,57 & 49,50 & 43,06 & 32,95 & 47,31 & & \\
\hline & 51,95 & 62,61 & 75,19 & 55,94 & 56,15 & & \\
\hline \multirow[t]{3}{*}{ Taxa de mortalidade por neoplasias } & 123,33 & 141,47 & 129,99 & 108,70 & 130,70 & \multirow[t]{3}{*}{1,94} & \multirow[t]{3}{*}{0,126} \\
\hline & 109,43 & 124,85 & 99,04 & 86,02 & 120,31 & & \\
\hline & 137,23 & 158,09 & 160,95 & 131,37 & 139,86 & & \\
\hline \multirow[t]{3}{*}{ Taxa de mortalidade por doenças circulatórias } & 227,03 & 313,98 & 291,54 & 276,45 & 282,18 & \multirow[t]{3}{*}{4,11} & \multirow[t]{3}{*}{0,008} \\
\hline & 202,95 & 280,31 & 221,83 & 222,68 & 261,49 & & \\
\hline & 251,09 & 347,65 & 361,25 & 330,21 & 302,87 & & \\
\hline \multirow[t]{3}{*}{ Taxa de mortalidade por doenças respiratórias } & 85,82 & 111,14 & 109,87 & 96,18 & 101,97 & \multirow[t]{3}{*}{2,45} & \multirow[t]{3}{*}{0,066} \\
\hline & 76,61 & 97,98 & 79,17 & 75,92 & 93,88 & & \\
\hline & 95,03 & 124,29 & 140,56 & 116,44 & 110,11 & & \\
\hline \multirow[t]{3}{*}{ Taxa de mortalidade por causas mal definidas } & 41,20 & 100,10 & 105,34 & 128,76 & 89,66 & \multirow[t]{3}{*}{17,19} & \multirow[t]{3}{*}{$<0,000$} \\
\hline & 32,88 & 87,63 & 77,24 & 95,72 & 79,92 & & \\
\hline & 49,51 & 112,57 & 133,45 & 161,80 & 99,41 & & \\
\hline \multirow[t]{3}{*}{ Taxa de mortalidade por causas externas } & 74,74 & 125,26 & 124,98 & 102,15 & 108,36 & \multirow[t]{3}{*}{7,57} & \multirow[t]{3}{*}{$<0,000$} \\
\hline & 62,80 & 110,24 & 95,21 & 77,63 & 98,76 & & \\
\hline & 86,67 & 140,28 & 154,74 & 126,66 & 117,95 & & \\
\hline
\end{tabular}

* Taxas de mortalidade ajustadas por faixa etária de cinco em cinco anos e mais de 70 anos de idade. IC = Intervalo de confiança.

Coeficientes de correlação bivariada de Pearson entre os indicadores sócio-econômicos e de mortalidade. Rio de Janeiro.

\begin{tabular}{|c|c|c|c|c|c|c|c|c|c|c|c|c|}
\hline Indicadores ${ }^{1}$ & T.M.Geral & $\begin{array}{l}\text { T.M. } \\
\text { Infecc. }\end{array}$ & $\begin{array}{l}\text { T.M. } \\
\text { Circ. }\end{array}$ & $\begin{array}{l}\text { T.M. } \\
\text { Neopl. }\end{array}$ & $\begin{array}{l}\text { T.M. } \\
\text { Resp. }\end{array}$ & $\begin{array}{l}\text { T.M. } \\
\text { Mal } \\
\text { Def. }\end{array}$ & $\begin{array}{l}\text { T.M. } \\
\text { Causa } \\
\text { Externa }\end{array}$ & $\begin{array}{l}\text { Proporção } \\
\text { de Casas }\end{array}$ & $\begin{array}{l}\text { P.D. } \\
\text { Subnorm. }\end{array}$ & $\begin{array}{l}\text { P.D. } \\
\text { com } \\
\text { Esgoto }\end{array}$ & $\begin{array}{l}\text { P.D. } \\
\text { Alugados }\end{array}$ & $\begin{array}{l}\text { P.C.F. } \\
\text { Renda } \\
<10 S M\end{array}$ \\
\hline T.M.Geral & 1,00 & $0,85^{\star *}$ & $0,97^{\star \star}$ & $0,91^{\star \star}$ & $0,94^{\star *}$ & $0,79 * *$ & $0,92^{\star *}$ & $0,22^{\star \star}$ & 0,07 & $-0,09$ & 0,06 & $-0,27^{\star \star *}$ \\
\hline T.M.Infecciosa & $0,85^{\star \star}$ & 1,00 & $0,78^{\star \star}$ & $0,76^{\star \star}$ & $0,79 * \star$ & 0,59 ** & $0,83^{\star \star}$ & 0,06 & $0,17^{\star}$ & 0,03 & $0,22^{\star \star}$ & $-0,20^{*}$ \\
\hline T.M.Circulatória & $0,97 * *$ & $0,78^{\star *}$ & 1,00 & $0,90^{\star *}$ & $0,94 * \star$ & $0,72^{* *}$ & $0,84^{\star \star}$ & $0,18^{*}$ & 0,01 & $-0,06$ & 0,07 & $-0,21 *$ \\
\hline T.M.Neoplasia & $0,91 * *$ & $0,76^{* *}$ & $0,90 * *$ & 1,00 & $0,89 * *$ & $0,55^{\star *}$ & $0,78^{\star *}$ & $-0,05$ & $-0,02$ & 0,09 & 0,12 & 0,02 \\
\hline T.M.Respiratória & $0,94 * \star$ & $0,79 * \star$ & $0,94 * \star$ & $0,89 * *$ & 1,00 & $0,65^{\star *}$ & $0,80^{\star \star}$ & 0,10 & 0,04 & $-0,05$ & 0,06 & $-0,14$ \\
\hline T.M.Mal Definida & $0,79 * \star$ & $0,59^{\star *}$ & $0,72^{\star *}$ & $0,55^{\star *}$ & $0,65^{\star *}$ & 1,00 & $0,75^{\star *}$ & $0,52^{\star \star}$ & 0,11 & $-0,35^{\star *}$ & $-0,12$ & $-0,52^{\star \star}$ \\
\hline T.M.Causa Externa & $0,92^{* *}$ & $0,83^{* *}$ & $0,84^{\star \star}$ & $0,78^{\star \star}$ & $0,80^{* *}$ & $0,75^{\star \star}$ & 1,00 & $0,25^{\star \star}$ & $0,17^{\star}$ & $-0,05$ & 0,08 & $-0,34^{\star \star}$ \\
\hline P.Casas & $0,22^{\star \star}$ & 0,06 & $0,18^{\star}$ & $-0,05$ & 0,10 & $0,52^{\star \star}$ & $0,25^{\star \star}$ & 1,00 & $0,41 * \star$ & $-0,56^{\star \star}$ & $-0,38^{\star \star}$ & $-0,77^{\star \star}$ \\
\hline P.D.Subnormais & 0,07 & $0,17^{\star}$ & 0,01 & $-0,02$ & 0,04 & 0,11 & $0,17^{\star}$ & $0,41^{\star *}$ & 1,00 & $-0,16$ & $-0,33^{\star *}$ & $-0,32^{\star \star}$ \\
\hline P.D.com Esgoto & $-0,09$ & 0,03 & $-0,06$ & 0,09 & $-0,05$ & $-0,35^{\star \star}$ & $-0,05$ & $-0,56^{\star *}$ & $-0,16$ & 1,00 & $0,49 * \star$ & 0,31 * \\
\hline P.D.Alugados & 0,06 & $0,22^{\star *}$ & 0,07 & 0,12 & 0,06 & $-0,12$ & 0,08 & $-0,38^{\star *}$ & $-0,33^{\star \star}$ & $0,49 * *$ & 1,00 & 0,01 \\
\hline P.C.F.Renda $<10 \mathrm{SM}$ & $\Lambda-0,27$ ** & $-0,20^{*}$ & $-0,21 *$ & 0,02 & $-0,14$ & $-0,52^{\star \star}$ & $-0,34^{\star *}$ & $-0,77^{\star *}$ & $-0,32^{\star \star}$ & $0,31 *$ & 0,01 & 1,00 \\
\hline
\end{tabular}

1 Estas correlações referem-se ao total de 144 bairros, devido à exclusão dos bairros com menos de 3.000 habitantes para o cálculo das taxas de mortalidade.

* Correlação significativa $p<0,01$, ** Correlação significativa $p<0,05$

T.M. =Taxa de Mortalidade Ajustada por Faixa Etária, P. = Proporção, P.D. = Proporção de Domicílios,

P.C.F. = Proporção de Chefes de Família, SM = Salários Mínimos; Infecc. = infecciosos; Circ. = circulatório

Resp. $=$ respiratórios; Mal Def. $=$ mal definidos; Subnorm. $=$ subnormais . 


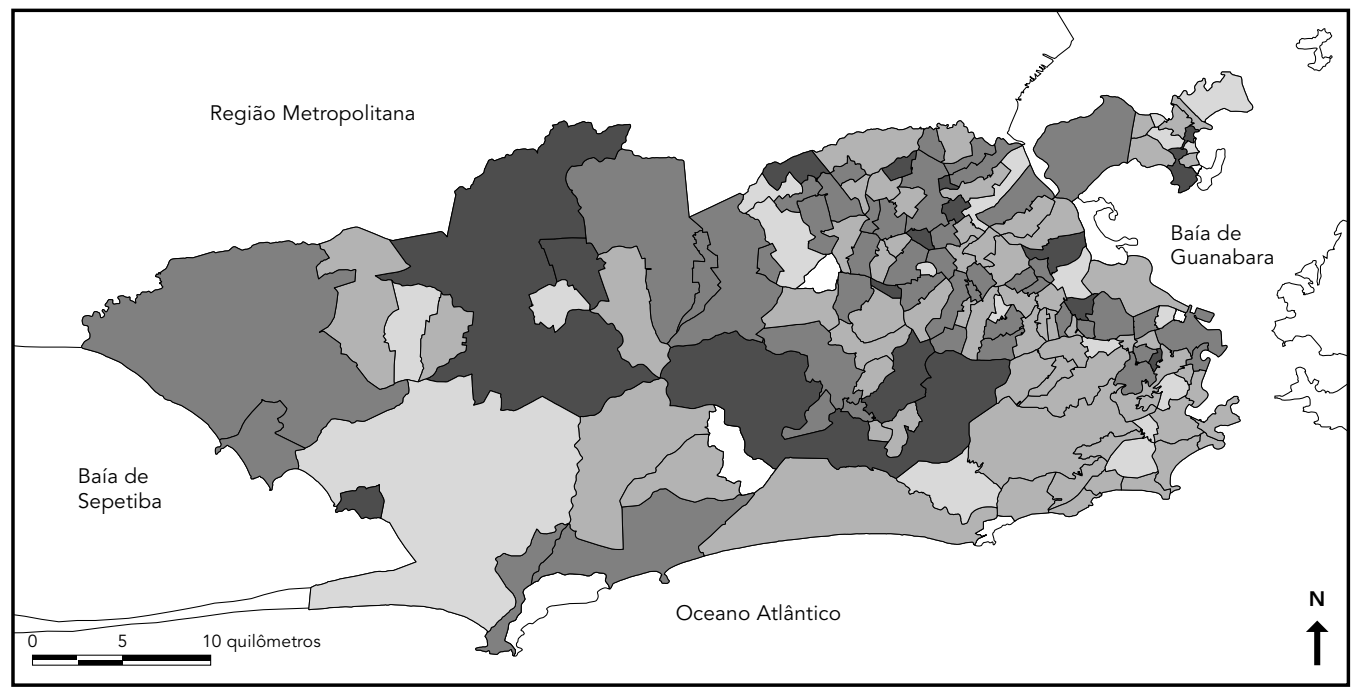

Taxa de mortalidade geral (100.000 habitantes)
$1.325,5$ a $2.618,2(16)$
913,3 a $1.325,5(46)$
501,1 a $913,3(63)$
126,1 a $501,1(19)$

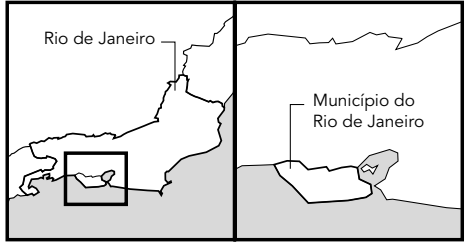

domicílios subnormais e inversa à renda, padrão que caracteriza as áreas de favela, mais desfavorecidas (Figura 3).

A taxa de mortalidade por causas mal definidas tem relação direta com a proporção de casas e inversa com a proporção de domicílios com esgoto ligado à rede geral e renda. Este padrão caracteriza as áreas menos ocupadas e com menor infra-estrutura urbana (Figura 4).

A taxa de mortalidade por doenças infecciosas tem relação direta com a proporção de setores subnormais e de domicílios alugados e inversa à renda.

As taxas de mortalidade por doenças respiratórias e neoplasias não apresentam correlação significativa com nenhum indicador sócioeconômico.

\section{Discussão}

Os resultados mostraram que a mortalidade geral ajustada, por doenças circulatórias, por causas mal definidas e por causas externas foram as variáveis com maior correlação com os indicadores sócio-econômicos na formação dos estratos, enquanto as taxas de mortalidade por doenças respiratórias e por neoplasias não apresentaram correlação significativa com nenhuma das variáveis analisadas.

As áreas que compõem o grupo A, com melhores indicadores sócio-econômicos, destacaram-se pelo seu perfil de baixa mortalidade. Por serem mais verticalizados e regulares, as condições de saneamento refletem a fiscalização municipal das construções. Este conjunto de bairros possui maior ocupação, com boa infra-estrutura urbana e de serviços. Em oposição a estas áreas, encontram-se os bairros da zona oeste, que compõem o grupo B, região mais distante do centro urbano da cidade, on- 


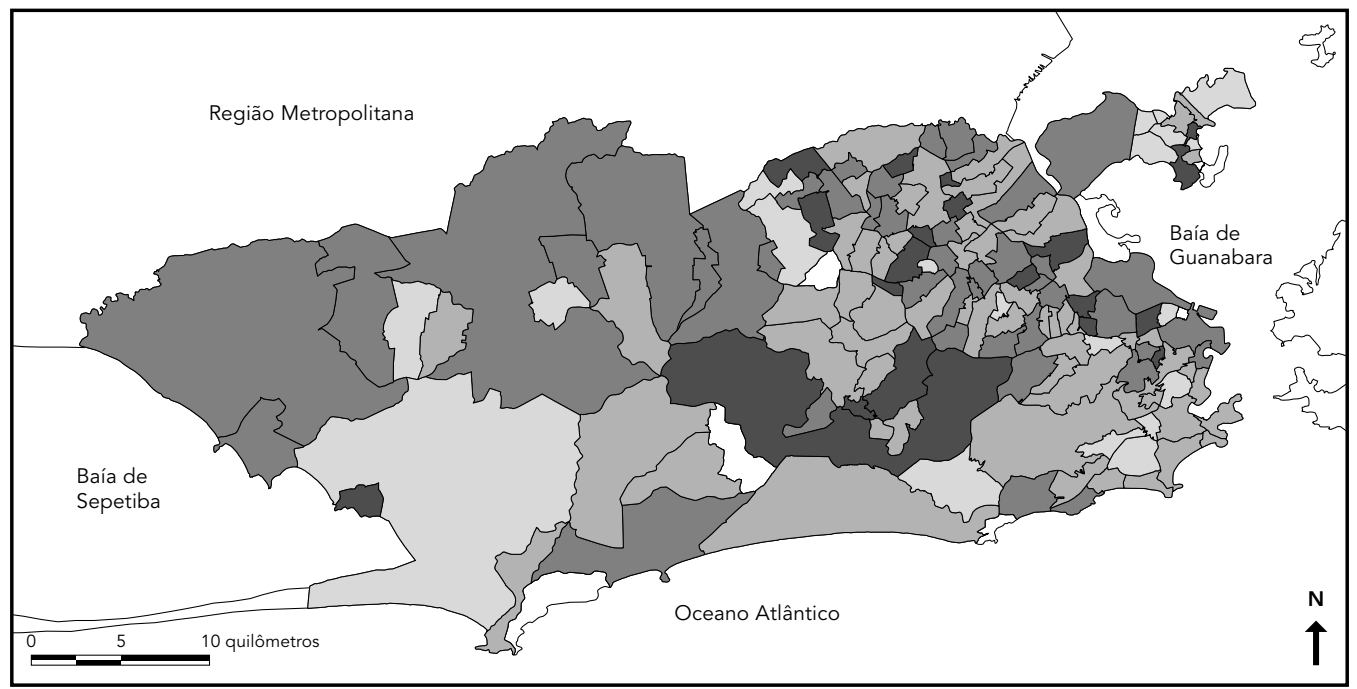

Taxa de mortalidade por causas externas (100.000 habitantes)
166,6 a $309,7(20)$
108,4 a $166,6(44)$
50,1 a $108,4(60)$
11,2 a $50,1(20)$

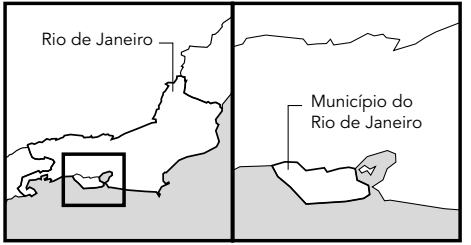

de a carência de serviços é maior. Esta carência expressa-se inclusive na baixa oferta de serviços de saúde, refletindo na menor especificação das causas de morte, fato que prejudica a análise mais detalhada dos componentes da mortalidade. Nas demais áreas, compostas pelos bairros de ocupação irregular (favelas) e de baixa renda, concentrados no grupo $\mathrm{C}$, e por bairros antigos, conhecidos como subúrbio carioca (Central e Leopoldina), concentrados no grupo D, sobressaíram as altas taxas de mortalidade por causas externas.

Independentemente da estrutura urbana dos bairros, a renda esteve fortemente correlacionada com as causas principais que compõem a mortalidade geral. A influência da renda fica mais evidente nas áreas de subúrbio (grupo D), onde, apesar da presença de maior estrutura de serviços, registraram-se altas taxas de mortalidade. Por sua vez, a violência, medida através da mortalidade por causas externas, está amplamente distribuída por toda a cidade, embora atingindo em menor escala os bairros que concentram moradores de alta renda (grupo A) e os bairros mais distantes na Zona Oeste (grupo B).

O uso dos diferentes indicadores sócio-econômicos permitiu análise mais detalhada das relações entre estes e cada componente da mortalidade geral. Importantes especificidades foram captadas de um modo que não seria possível se fossem utilizados indicadores sintéticos de condições de vida. Apesar dos indicadores sintéticos serem úteis para comparação entre municípios, estados e países por refletirem condições gerais de vida, a complexidade dos eventos de saúde em escalas locais necessita de indicadores que discriminem as heterogeneidades dos fatores sociais e ambientais que trazem risco à saúde.

A análise espacial em saúde, baseada em dados secundários, traz problemas comuns a outros sistemas de informação, como os relacionados à qualidade dos dados e problemas 


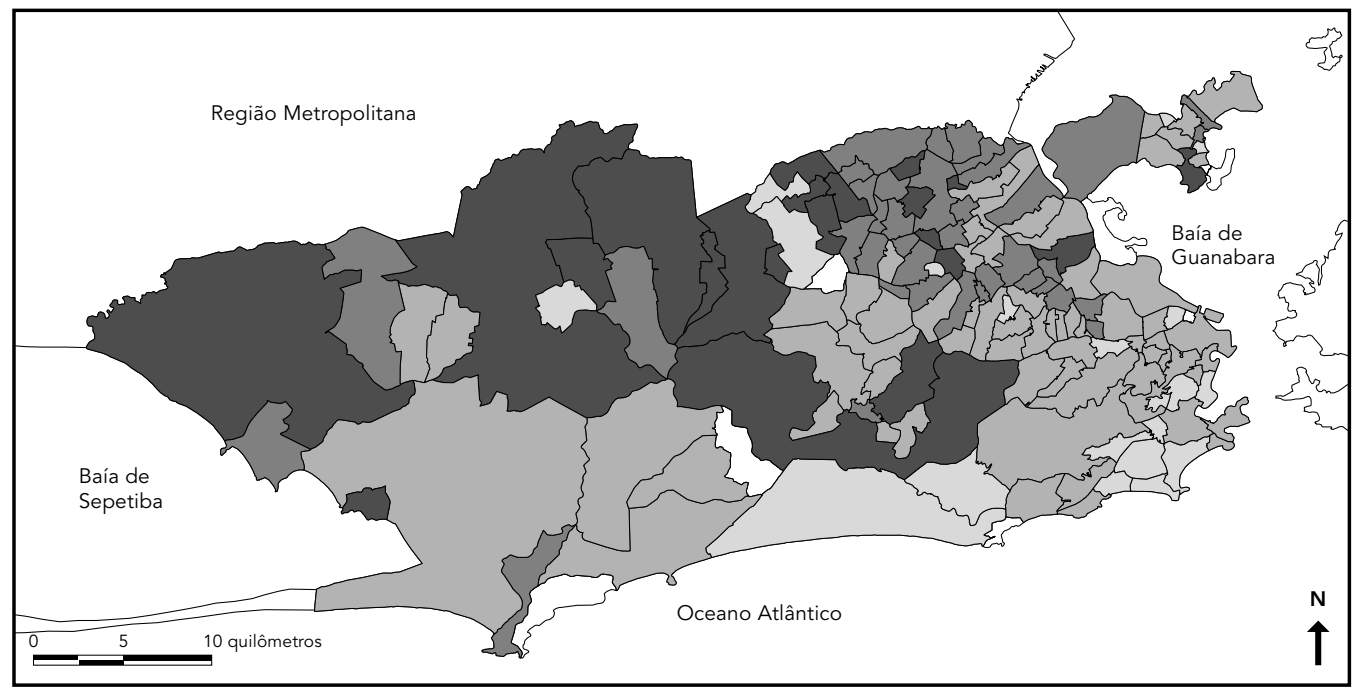

Taxas de mortalidade por causas mal definidas (100.000 habitantes)
148,8 a $295,1(21)$
89,7 a $148,8(38)$
30,5 a $89,7(65)$
10,7 a $30,5(20)$

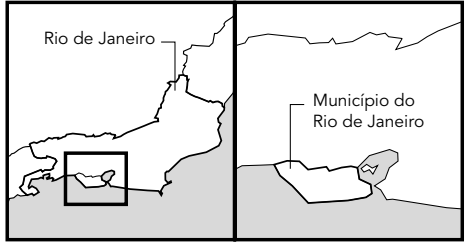

ligados à variável utilizada para o georreferenciamento. Dentre os óbitos analisados, apenas $2,34 \%$ tiveram bairro de residência ignorado, garantindo bom preenchimento dos registros. Por sua vez, o padrão de distribuição espacial da mortalidade é influenciado pelos erros de classificação do evento nas diversas áreas da cidade. Advindo de um processamento de dados não estruturado para a captação dos endereços residenciais segundo uma padronização da totalidade dos logradouros da cidade (ruas, avenidas, praças, travessas etc.) pela sua própria inexistência, este erro pode causar pequena distorção na classificação. Isto pode ocorrer em duas situações principais: a referência ao bairro vizinho, quando um bairro pequeno é vizinho de um bairro pólo de atração (Corrêa, 1986), fazendo com que bairros mais conhecidos pela população sejam mais citados do que outros. Nesta situação de provável sobreenumeração de mortes encontram-se os bairros de Jacarepaguá e Campo Grande. Outra situação é a referência de um endereço de residência em locais próximos às áreas cobertas por determinados serviços de saúde como, por exemplo, no caso de pacientes com neoplasia em busca de assistência (já que o endereço que consta na declaração de óbito é geralmente retirado dos prontuários hospitalares).

Embora a taxa de mortalidade geral represente a expressão da totalidade dos óbitos ocorridos em pessoas residentes na Cidade do Rio de Janeiro, as taxas referentes aos capítulos de causas específicas de mortes não violentas, as mortes também conhecidas como naturais, tendem a sofrer problemas de subenumeração em função das altas freqüências de causas mal definidas registradas na cidade. Como a ocorrência de mortes não violentas de causas mal definidas não segue padrão homogêneo na sua distribuição espacial, a influência de seu efeito sobre o conjunto de mortes de causas específicas também ocorrerá de forma a causar mais perda de informação nas áreas da cidade com 
maiores problemas de assistência médica. $\mathrm{Ou}$ seja, o efeito da ocorrência das mortes mal definidas não se dá com a mesma intensidade nos bairros da cidade, prejudicando as taxas específicas dos demais capítulos de causas nestas regiões, com a mesma intensidade daquela de sua própria freqüência. Esta característica atinge todas as causas de morte, principalmente os grupos de causas mais freqüentes, tais como as doenças do aparelho circulatório, as neoplasias e as doenças do aparelho respiratório.

Os achados empíricos deste estudo confirmam a participação das condições de vida na determinação do padrão de mortalidade da população carioca, em consonância com os estudos anteriormente realizados que discutem a participação das desigualdades sociais nos desfechos de saúde da população (Silva et al., 1999; Szwarcwald et al., 1999). Por sua vez, eles também evidenciam a necessidade de redirecionamento das políticas públicas, buscando maior adequação dos investimentos municipais nas áreas mais carentes de infra-estrutura urbana através da melhoria da oferta de serviços públicos e implementação de programas sociais específicos com vistas a reduzir as grandes desigualdades existentes na cidade do Rio de Janeiro.

Estudos como este evidenciam a enorme contribuição que pode ser dada pelo sistema de informações sobre mortalidade, quando se analisa suas características segundo a distribuição espacial dos eventos, para caracterização da situação de saúde de microáreas. Estudos deste tipo também podem ser úteis para a avaliação dos resultados de políticas públicas, em particular, aquelas com impacto na saúde, em outras cidades brasileiras, a médio e longo prazo.

\section{Agradecimentos}

Agradecemos o apoio ao projeto Uso de Indicadores Urbanos de Condições de Vida e suas Relações com Riscos Diferenciais de Mortalidade: O Espaço de Viver e Morrer, concedido na forma de bolsa de pesquisador visitante através do convênio Fundação Oswaldo Cruz/Fundação de Amparo à Pesquisa do Estado do Rio de Janeiro.

Agradecemos também ao pesquisador Christovam Barcellos (Departamento de Informação em Saúde/Centro de Informação Científica e Tecnológica/Fundação Oswaldo Cruz) pela valorosa troca de idéias.

\section{Referências}

AKERMAN, M.; STEPHENS, C.; CAMPANARIO, P. \& MAIA, P. B., 1994. Saúde e meio ambiente: Uma análise de diferenciais Intra-urbanos enfocando o município de São Paulo, Brasil. Revista de Saúde Pública, 28:320-325.

BARCELLOS, C., 2000. Organização espacial, saúde e qualidade de vida. In: Seminário Nacional Saúde e Ambiente no Processo de Desenvolvimento (Fundação Oswaldo Cruz, org.), Série Fiocruz: Eventos Científicos 2, pp. 27-34, Rio de Janeiro: Fundação Oswaldo Cruz.

BARCELLOS, C. \& BASTOS, F. I., 1996. Geoprocessamento, ambiente e saúde: Uma união possível? Cadernos de Saúde Pública, 12:389-397. 
BARCELLOS, C. \& SANTOS, S. M., 1997. Colocando dados no mapa: A escolha da unidade de agregação e integração de bases de dados em saúde e ambiente através do geoprocessamento. Informe Epidemiológico do SUS, 6:21-29.

CARVALHO, M. S.; CRUZ, O. G. \& NOBRE, F. F., 1997. Perfil de risco: Método multivariado e classificação sócio-econômica de microáreas urbanas - Os setores censitários da região metropolitana do Rio de Janeiro. Cadernos de Saúde Pública, 13:635-645.

CORRÊA, R. L., 1986. Região e Organização Espacial. São Paulo: Editora Ática.

DI VILLAROSA, F. N.; TASCA, R. \& FERNANDES, R. V., 1990. Análise da situação sócio-sanitária, microlocalização e participação no distrito sanitário de Pau da Lima, Salvador. Revista Bahiana de Saúde Pública, 17:7-14.

D’ORSI, E., 1996. Perfil de Nascimentos e Condições Sócio-econômicas no Município do Rio de Janeiro: Uma Análise Espacial. Dissertação de Mestrado, Rio de Janeiro: Escola de Saúde Pública, Fundação Oswaldo Cruz.

D’ORSI, E. \& CARVALHO, M. S., 1998. Perfil de nascimentos no Município do Rio de Janeiro: Uma análise espacial. Cadernos de Saúde Pública, 14: 367379.

FREITAS, E. D.; PAIM, J. S.; SILVA, L. M. V. \& COSTA, M. C. N., 2000. Evolução e distribuição espacial da mortalidade por causas externas em Salvador. Cadernos de Saúde Pública, 16:109-118.

HARTIGAN, J. Á., 1975. Clustering Algorithms. New York: John Wiley \& Sons Inc.

IBGE (Fundação Instituto Brasileiro de Geografia e Estatística), 1994. Censo Demográfico, Brasil, 1991. Rio de Janeiro: IBGE.

IBGE (Fundação Instituto Brasileiro de Geografia e Estatística), 1998. Contagem da População, 1996. v. I e II. Rio de Janeiro: IBGE.

LIMA, M. L. C. \& XIMENES, R., 1998. Violência e morte: Diferenciais da mortalidade por causas externas no espaço urbano do Recife, 1991. Cadernos de Saúde Pública, 14:829-840.

MAGUIRRE, D. J.; GOODCHILD, M. F. \& RHIND, D. W., 1991. Geographical Information Systems: Principles and Applications. London: Longman Scientific \& Technical.
NORONHA, C. P.; IOZZI-SILVA, R. \& THEME FILHA, M. M., 1998. Diferenciais internos de mortalidade na cidade do Rio de Janeiro. In: IV Congresso Brasileiro de Epidemiologia, Resumos, p. 336. Rio de Janeiro: ABRASCO.

OMS (Organização Mundial de Saúde), 1995. Classificação Estatística Internacional de Doenças e Problemas Relacionados à Saúde, 10a Revisão. v. 1. São Paulo: Centro Colaborador da OMS para Classificação de Doenças em Português.

PAIM, J. S.; COSTA, M. C. N.; MASCARENHAS, J. C. S. \& SILVA, L. M. V., 1999. Distribuição espacial da violência: Mortalidade por causas externas em de Salvador (Bahia), Brasil. Revista Panamericana de Salud Publica, 6: 321-332.

ROJAS, L. I.; BARCELLOS, C. \& PEITER, P., 1999. Utilização de mapas no campo da epidemiologia no Brasil. Informe Epidemiológico do SUS, 8:25-35.

SILVA, L. M.; PAIM, J. S. \& COSTA, M. C. N., 1999. Desigualdades na mortalidade, espaço e estratos sociais. Revista de Saúde Pública, 33:6-97.

SMS-RJ (Secretaria Municipal de Saúde, Rio de Janeiro), 1999. Sistema de Informações sobre Mortalidade no Município do Rio de Janeiro, 1997-1998. Rio de Janeiro: Gerência de Informações Epidemiológicas, Coordenação de Programas de Epidemiologia, Superintendência de Saúde Coletiva, Secretaria Municipal de Saúde.

SOUZA, E. R.; ASSIS, S. G. \& SILVA, C. M. F. P., 1997. Violência no Município do Rio de Janeiro: Áreas de risco e tendências da mortalidade entre adolescentes de 10 a 19 anos. Revista Panamericana de Salud Publica, 1:389-397.

SPSS INCORPORATION, 1997. SPSS for Windows. Statiscal Package for the Social Sciences. Release 8.0. Chicago: SPSS Inc.

SZWARCWALD, C. L.; BASTOS, F. I.; ESTEVES, M. A. P.; ANDRADE, C. L. T.; PAEZ, M. S.; MEDICI, E. V. \& DERICO, M., 1999. Desigualdade de renda e situação de saúde: O caso do Rio de Janeiro. Cadernos de Saúde Pública, 15:15-28. 